\title{
Recombinant Human Endostatin Endostar Inhibits Tumor Growth and Metastasis in a Mouse Xenograft Model of Colon Cancer
}

\author{
Yitao Jia • Min Liu • Wangang Huang • Zhenbao Wang • Yutong He • Jianhua Wu • \\ Shuguang Ren • Yingchao Ju • Ruichao Geng • Zhongxin Li
}

Received: 7 December 2010 / Accepted: 1 August 2011 /Published online: 22 September 2011

(C) The Author(s) 2011. This article is published with open access at Springerlink.com

\begin{abstract}
To investigate the effects of recombinant human endostatin Endostar on metastasis and angiogenesis and lymphangiogenesis of colorectal cancer cells in a mouse xenograft model. Colon cancer cells SW620 were injected subcutaneously into the left hind flank of nude mice to establish mouse xenograft models. The mice were treated with normal saline or Endostar subcutaneously every other day. The growth and lymph node metastasis of tumor cells, angiogenesis and lymphangiogenesis in tumor tissue were detected. Apoptosis and cell cycle distribution were studied by flow cytometry. The expression of VEGF-A, $-\mathrm{C}$, or -D in SW620 cells was determined by immunoblotting assays. Endostar inhibited tumor growth and the rate of lymph node metastasis $(P<0.01)$. The density of blood vessels in or
\end{abstract}

\footnotetext{
Y. Jia

Department of Oncology, Hebei General Hospital,

Shijiazhuang, Hebei, China

e-mail: jiayitao1970@yahoo.cn

M. Liu

Geriatrics Key Laboratory, Hebei General Hospital,

Shijiazhuang, Hebei, China

e-mail: minmin2583@126.com

W. Huang $\cdot$ Z. Wang $\cdot$ R. Geng $\cdot$ Z. Li $(\bowtie)$

Second Department of Surgery, the Forth Hospital, Hebei Medical University,

Shijiazhuang, Hebei, China

e-mail: lizhongxin99@yahoo.com.cn

W. Huang

e-mail: wanganghuang@163.com

Z. Wang

e-mail: wang.zhenbao88@gmail.com

R. Geng

e-mail: grc0303@sina.com
}

around the tumor area was $12.27 \pm 1.21$ and $22.25 \pm 2.69$ per field in Endostar-treated mice and controls $(P<0.05)$, respectively. Endostar also decreased the density of lymphatic vessels in tumor tissues $(7.84 \pm 0.81$ vs. $13.83 \pm 1.08$, $P<0.05)$. Endostar suppresses angiogenesis and lymphangiogenesis in the lymph nodes with metastases, simultaneously. The expression of VEGF-A, -C and -D in SW620 cells treated with Endostar was substantially lower than that of controls. Endostar inhibited growth and lymph node metastasis of colon cancer cells by inhibiting angiogenesis and lymphangiogenesis in a mouse xenograft model of colon cancer.

Keywords Recombinant human endostatin - Colorectal cancer $\cdot$ Metastasis $\cdot$ Angiogenesis $\cdot$ Lymphangiogenesis

Y. He

Cancer Institute of Hebei Province,

Shijiazhuang, Hebei, China

e-mail: hytong69@yahoo.com

J. $\mathrm{Wu} \cdot \mathrm{S}$. Ren $\cdot$ Y. Ju

Centre of Animal Experiment of the Fourth Hospital of Hebei

Medical University,

Shijiazhuang, Hebei, China

J. Wu

e-mail: WuJH-890@126.com

S. Ren

e-mail: rsg9802@126.com

Y. Ju

e-mail: juyingchao@163.com 


\section{Introduction}

Colorectal cancer is the third most frequent cancer worldwide after breast and lung cancers and is also one of the most common causes of cancer death worldwide, ranking second as the most fatal malignancy in developed countries. The impact of this disease is staggering worldwide: the annual incidence of colorectal cancer has been on the rise in many countries except the USA [1]. Conventional therapies including surgery, chemotherapy or radiotherapy have been proven to be relatively ineffective [2]. The main reasons for these unsuccessful therapies are regional lymph node and/or distal metastasis of colorectal cancer cells [3]. Approximately one quarter of colorectal cancer patients present with metastatic disease at the time of diagnosis and their five-year survival rates are approximately $10 \%$ [4]. However, the mechanisms underlying this malignancy still remain largely unknown. For this reason, elucidating cellular factors involved in the metastatic cascade is a key goal for developing therapeutic agents to reduce colorectal cancer-associated mortality.

Dependence of tumor growth and metastasis on blood vessels makes angiogenesis a fundamental hallmark of cancer. Angiogenesis occurs in the microenvironment of many solid tumors including colorectal cancer [5] and is driven by multiple factors such as basic fibroblast growth factor and vascular endothelial growth factor (VEGF)-A, which are essential for the growth and metastasis of colorectal cancer [5, 6]. For this reason, anti-angiogenic therapy has emerged as a therapeutic option for malignant diseases. Agents targeting directly at VEGF-A, such as bevacizumab, have become part of the standard regimen for metastatic colorectal cancer. Several researches indicated an advantage in favor of the addition of bevacizumab to chemotherapy in terms of overall survival, progression-free survival and response rate [7]. However, the development of bevacizumab-resistant tumors has become more common since its integration into the treatment of cancers [8]. Furthermore, some side effects attributable to bevacizumab therapy were reported to date [9].

Lymphangiogenesis, in addition to angiogenesis, also occurs in colorectal cancer $[10,11]$. The density of lymphatic vessels in or surrounding tumors is closely associated with lymph node metastasis and prognosis of colorectal cancer patients $[12,13]$. The best validated molecular signaling system for tumor lymphangiogenesis involves VEGF-C and VEGF-D, which induce the growth of lymphatic vessels by activating VEGF receptor-3 (VEGFR-3) on the surface of lymphatic endothelial cells [14]. Thus, anti-lymphangiogenesis therapy may be another attractive anticancer treatment strategy $[15,16]$. While antilymphangiogenesis is not tested clinically yet, tailored treatments consisting of combinations of chemotherapy, anti-angiogenesis, and anti-lymphangiogenesis agents will hopefully improve patient outcomes. Interestingly, endostatin, a broad-spectrum endogenous inhibitor of angiogenesis, which inhibits the proliferation of endothelial cells, inhibits both lymphangiogenesis in tumors and lymph node metastasis of tumor cells simultaneously [17-19].

Previous studies have suggested that plasma endostatin levels are elevated in colorectal cancer patients with liver metastases [20]. Endostatin inhibited not only colon cancer growth in vitro and in vivo, but also reduced colorectal cancer cell adhesion in the liver sinusoids during the very early phases of metastasis formation in a liver metastasis mouse model [21, 22]. Because endostatin is naturally occurring, and its half-life is short, there have been numerous attempts to modify endostatin, with some successes. Endostar, a novel recombinant human endostatin, is purified in E. coli with an additional nine-amino acid sequence that forms a His-tag structure. Endostar has been approved by the State Food and Drug Administration of China (SFDA) for the treatment of non-small cell lung cancer in 2005 [23]. More recently, it has been shown that Endostar combined with oxaliplatin inhibited growth and induced apoptosis in a human colorectal carcinoma model [24]. However, it is not clear whether Endostar inhibits the lymphangiogenesis or metastasis of colorectal cancer.

In the present study, we used a colon cancer xenograft mouse model to evaluate the effect of Endostar on tumor progression, angiogenesis or lymphangiogenesis of colorectal cancer. We find that Endostar inhibited both the growth and lymph node metastasis of colorectal cancer by inhibiting the angiogenesis and lymphangiogenesis in a mouse xenograft model of colon cancer. Our current work provides a new prospect for targeted therapy of colorectal cancer.

\section{Materials and Methods}

\section{Cell Line and Animals}

Luciferase-expressing human colon cancer cell line SW620, a gift from Longmed Inc. (United States (US)), was maintained in Minimum Essential Medium (Gibco, Grand Island, NY) supplemented with $10 \%$ fetal bovine serum at $37^{\circ} \mathrm{C}$ in a humidified atmosphere containing $5 \% \mathrm{CO} 2$.

Five to 6 week old male nude mice (BALB/c nu/nu), which were purchased from the Institute of Laboratory Animal Science, Chinese Academy of Medical Sciences, Beijing, China, were housed in environmentally controlled conditions $\left(22^{\circ} \mathrm{C}\right.$, a $12-\mathrm{h}$ light/dark cycle with the light cycle from 6:00 to 18:00 and the dark cycle from 18:00 to 6:00) with ad libitum access to standard laboratory chow. The study protocol was approved by the local Institution Review Board and animal experiments were conducted in accordance with the guideline of the local Institutional Animal Care and Use Committee, 
which has been accredited by the Association for Assessment and Accreditation of Laboratory Animal Care International.

\section{Mouse Xenograft Study}

SW620 cells were rendered into single-cell suspensions and 5 to $6 \times 10^{6}$ cells in $200 \mu \mathrm{L}$ serum-free medium were injected subcutaneously into the left hind flank of BALB/c mice. Tumors were allowed to grow untreated until they reached $7 \mathrm{~mm}$ in diameter, and then the mice were randomly divided into two groups $(n=9)$. Group 1 (control group) was treated with normal saline (NS) (200 $\mu \mathrm{L}$ for each mouse) by subcutaneous injection around the tumor every other day. Group 2 was treated with Endostar (purchased from Simcere Pharmaceutical Co., Jiangsu, China) at $10 \mathrm{mg} / \mathrm{kg}$ by subcutaneous injection around the tumor every other day. All these treatments were maintained for four weeks. Tumor growth and metastasis were monitored using the Xenogen IVIS Imaging System (Xenogen, US). The mice underwent initial imaging at one week post inoculation when the tumor length reached approximately $7 \mathrm{~mm}$ in length. For in vivo imaging, mice were administered D-luciferin $(150 \mathrm{mg} / \mathrm{kg})$ (Promega, Madison, WI) by intraperitoneal injection. Fifteen min later, photons from the whole animal body were counted using the Xenogen IVIS system. Imaging was performed once weekly. Data were analyzed using the Living Image 2.50 Software (Xenogen). In addition, one week after the completion of the treatment regimen, all mice were weighed and then sacrificed by application of chloral hydrate anesthesia. A complete autopsy, including lymph node dissection, was performed evaluated histopathologically for the presence of metastases. The samples were routinely sectioned and stained with hematoxylin and eosin (H\&E) and evaluated by two pathologists in a double-blinded manner.

Determination of Blood Vessel and Lymph Vessel Densities

Tissue sections were stained with anti-CD31 antibody (rat anti-mouse monoclonal antibody, 1:200, eBioscience, US) and anti-LYVE-1 antibody (rabbit anti-mouse polyclonal antibody, 1:200, Upstate, US). Biotinylated anti-rat or rabbit antibodies (Beijing Zhongshan Golden Bridge Biological Technology CO., Beijing, China) were used as secondary antibodies. Three or 4 fields per tumor or lymph node with metastases were scored for microvessel and lymph vessel elements at $\times 200$ magnifications by two doubleblinded observers, and microvessel and lymph vessel densities in tumor or positive lymph nodes were calculated.

\section{Western Blotting}

SW620 cells were treated with $10 \mu \mathrm{g} / \mathrm{mL}$ Endostar and $24 \mathrm{~h}$ post treatment cellular lysates were prepared as previously described [12]. Immunoblotting study was performed as previously depicted [13] and the following antibodies were used: anti-VEGF-A (rabbit anti-human polyclonal antibody, 1:100, Santa Cruz, US), VEGF-C (goat anti-human monoclonal antibody, 1:100, BioVision, US), VEGF-D (mouse anti-human monoclonal antibody, 1:100; Santa Cruz, US) or $\beta$-actin (Santa Cruz Biotechnology) antibodies. The immunoblots were analyzed and quantified by MCID imaging software (Imaging Research Inc., St. Catharines, Ontario, Canada). The expression of VEGF-A, -C and $-\mathrm{D}$ was normalized against $\beta$-actin.

\section{Statistical Analysis}

Differences in metastasis formation between groups were analyzed using the $\chi^{2}$ test and confirmed with Fisher's exact test. The other data were expressed as the mean \pm standard deviation $(\overline{\mathrm{x}} \pm \mathrm{s})$. One-way analysis of variance (ANOVA) was used to assess the statistical significance of differences between groups using SPSS 15.0 software. $P<0.05$ was considered statistically significant.

\section{Results}

During the treatment regimen, one mouse in the control group died. All of the other mice survived until the experiment was completed.

\section{Endostar Inhibits Metastasis of Tumor Cells to Regional Lymph Nodes}

To evaluate the effect of Endostar on regional lymph node metastasis of colorectal cancer cells, we established a mouse xenograft model of colorectal cancer using the human colon cancer cell line SW620, which has a high frequency of lymph node metastasis. Using the Xenogen IVIS Imaging System, we observed early indication of metastasis to the lymph nodes in the axilla and groin region 4 weeks after inoculation of SW620 cells into the control mice (Fig. 1a). On the other hand, we detected photons in lymph nodes in the axilla and groin region in only 3 out 9 of Endostar-treated mice (Fig. 1b). Quantification of the amount of photons emitted by luciferase expressing tumors showed that, compared with that of the control group, the amount of photons emitted by luciferase expressing tumors in mice receiving Endostar was markedly decreased from week 3 post tumor implantation (Fig. 1c) $(P<0.05)$. Furthermore, examination of lymph nodes in the axilla and groin region of sacrificed mice at week 6 post tumor cell inoculation revealed that tumor cells metastasized to the lymph nodes in $33.3 \%$ (3/9) of Endostar-treated mice while all the mice in the control group showed metastasis to 


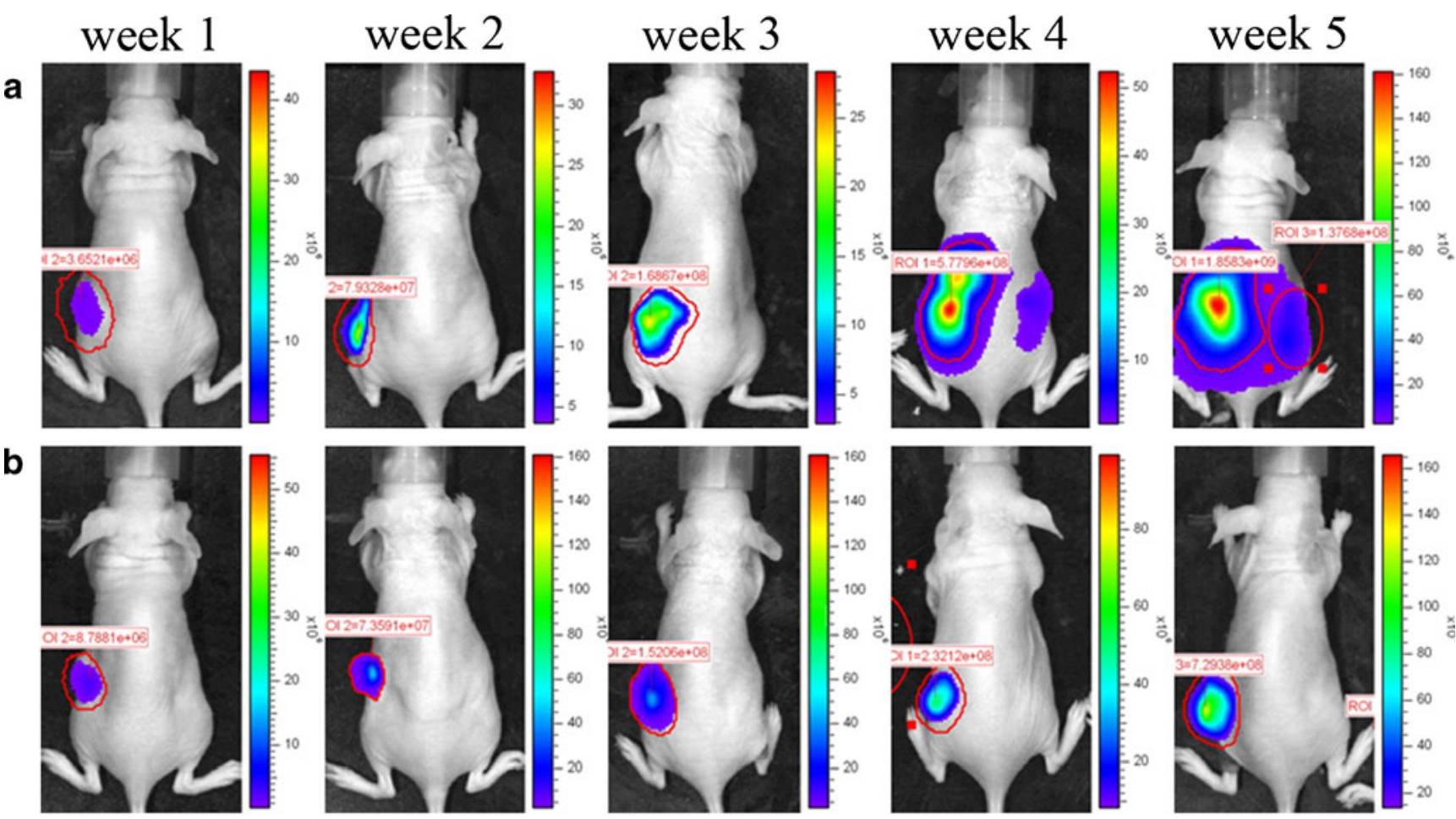

C

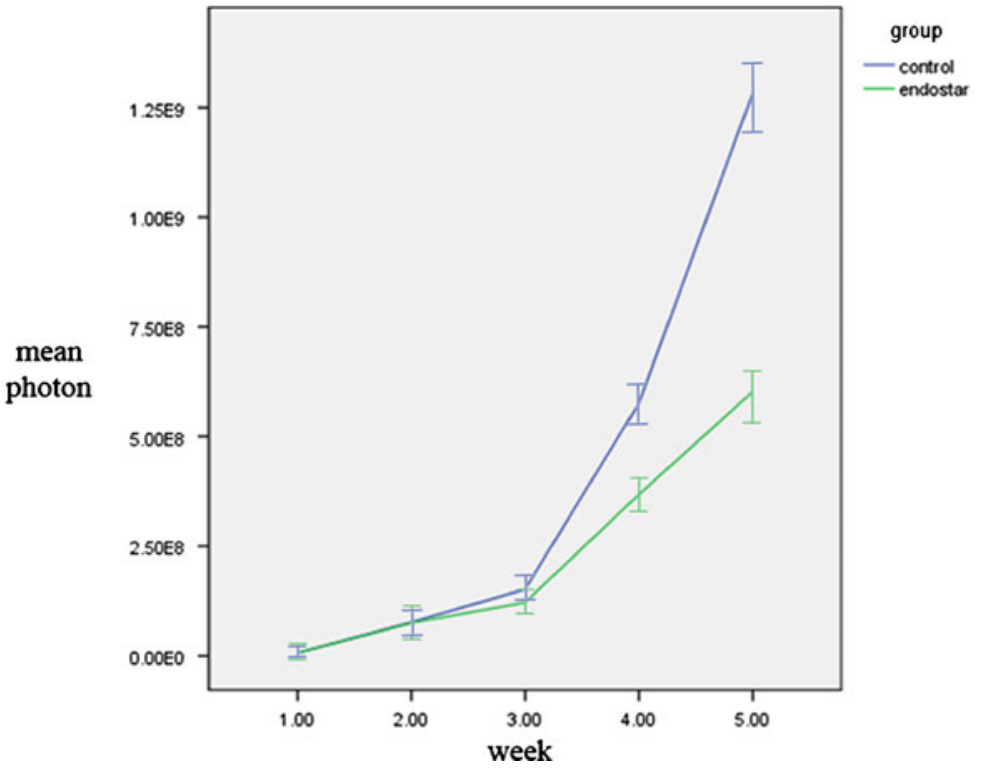

Fig. 1 The activity and metastasis of tumor cells as evaluated by Xenogen IVIS Imaging System in vivo (a): the control group; (b): the Endostar group; (c): Growth curve of tumors on the nude mice model of human colon cancer. Tumor growth was inhibited significantly in the Endostar group than in the control group $(P<0.05)$

positive in Endostar-treated group animals. The average number of metastatic lymph nodes per node-positive mouse in the Endostar-treated group was significantly smaller than that in the control group $(1.33 \pm 0.57$ vs $2.63 \pm 0.7, P<0.05)$ (Table 1). These results indicated that Endostar could suppress the metastasis of colon cancer cells in mice bearing human colon cancer xenograft. 

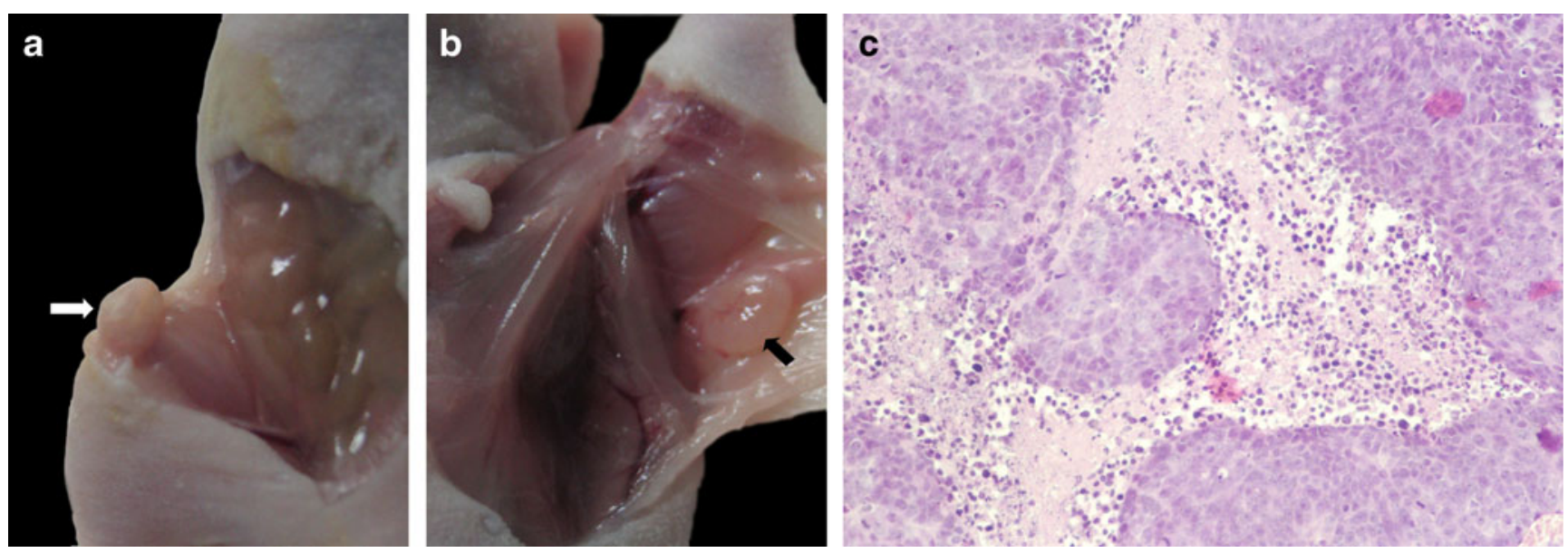

Fig. 2 Gross and histological appearance of primary SW620 colon cancer and lymph node metastasis. Grossly enlarged regional lymph node of the inguina (a) and axilla (b) (arrow); H\&E-staining of the metastatic tumor in the lymph node (C) $(\times 100)$

Endostar Inhibits Blood and Lymph Vessel Formation In Vivo

To investigate the effect of Endostar on tumor neoangiogenesis, we examined the tissue sections by immunohistochemical staining with anti-CD31antibody to visualize tumor blood vessels in the control mice (Fig. 3a) and in the mice bearing human colon cancer xenograft treated with Endostar (Fig. 3b). The average number of blood vessels in the tumor area per visual field was $22.25 \pm 2.69$ in the control xenografts and $12.27 \pm 1.21$ in the xenograft treated with Endostar group and control group, respectively $(P<0.05)$ (Fig. 3e). We further examined the number of lymphatic vessels by immunohistochemical staining with anti-LYVE-1 antibodies to visualize tumor lymphatic vessels in the control mice and in the mice bearing human colon cancer xenograft treated with Endostar. The density of lymphatic vessels in the tumor area per visual field was $13.83 \pm 1.08$ in the control group and $7.84 \pm 0.81$ in the xenograft treated with Endostar group, respectively $(P<0.05)$ (Fig. 3f). Several expanded lymphatic vessels were also observed within or around the tumors in the control group and tumor thrombus were observed in some of those expanded lymphatic vessels. In contrast, most LYVE-1 positive vessels were stripe-like in mice treated with Endostar (Fig. 3c and d). Our data demonstrated that Endostar could inhibit both angiogenesis and lymphangiogenesis in a mouse xenograft model of colon cancer.

The vessel numbers in the positive lymph nodes were also measured. The density of blood vessels in Endostar-treat group were remarkably reduced compared to the control group $(17.24 \pm 2.31$ vs $9.63 \pm 1.87, P<0.05)$. A significant reduction in intratumor lymphatic vessels density was observed in mice that received Endostar therapy when compared with animals in the control group $(8.43 \pm 1.25$ vs $3.54 \pm 1.07, P<0.05)$, suggested that Endostar can be functional within the whole body.

Expression of VEGF-A, VEGF-C and VEGF-D in SW620 Cells

VEGF-A, -C and -D expression has been correlated with the growth of lymphatic vessels. In order to unveil how Endostar inhibited lymphangiogenesis, we investigated the effect of Endostar on the expression of VEGF-A, -C and D in SW620 cells by immunoblotting using anti-VEGF antibodies. We found that Endostar treatment noticeably decreased the levels of VEGF-A, -C and -D. The level of VEGF-A in SW620 cells treated with Endostar was 2.37fold lower than that of controls and the level of VEGF-C and VEGF-D in SW620 cells treated with Endostar was

Table 1 Effect of Endostar on lymph node metastasis in mouse xenograft model of colorectal cancer

\begin{tabular}{llll}
\hline & No. of mice & No. of node- positive mice ${ }^{\mathrm{a}}$ & No. of positive lymph nodes $^{\mathrm{b}}$ \\
\hline Control & 8 & $8(100 \%)$ & $2.63 \pm 0.7(n=8)$ \\
Endostar & 9 & $3(33.3 \%)$ & $1.33 \pm 0.57(n=3)$ \\
$P$ value & & $<0.01$ & $<0.05$ \\
\hline
\end{tabular}

${ }^{\text {a }}$ Metastasis to the regional lymph nodes was detected by histological examination.

${ }^{\mathrm{b}}$ Mean \pm SEM of the number of metastatic lymph nodes per mouse. 

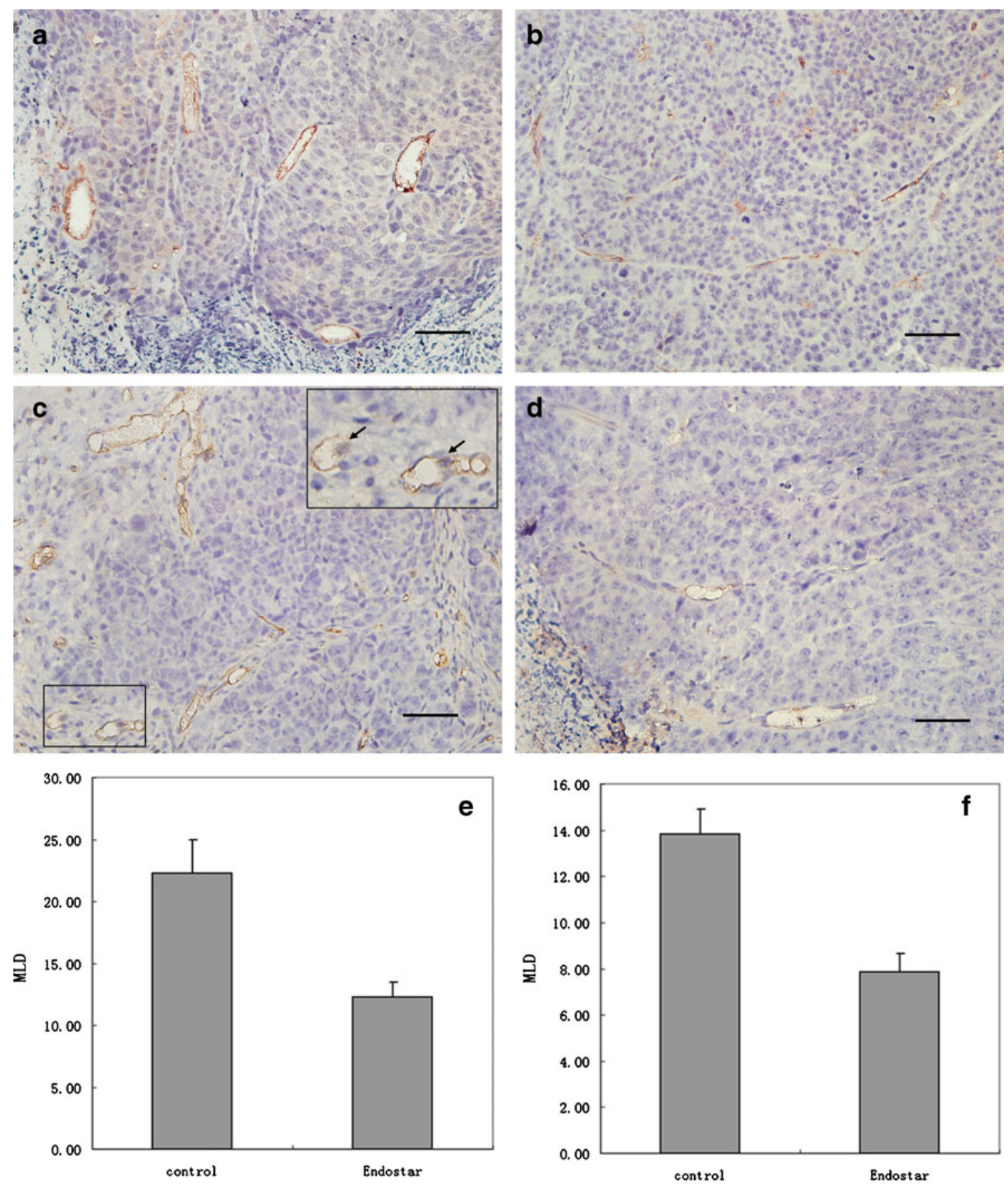

Fig. 3 CD31-positive blood vessels and LYVE-1-positive lymphatic vessels in tumor tissue. a: Several expanded blood vessels were within the tumors in the control mice $(\times 200)$. b: The blood vessels were stripe like in Endostar-treated mice $(\times 200)$. c: Several expanded lymphatic vessels with tumor thrombus (arrows) were also observed within and around the tumors in the control mice $(\times 200)$. d: Most

1.18-fold and 1.49-fold lower than that of controls, respectively (Fig. 4). These results suggested that Endostar could inhibit the angiogenesis and lymphangiogenesis of human colon cancer cells by downregulating the expression of VEGF-A,-C,-D.
LYVE-1 positive vessels were stripe-like in mice treated with Endostar $(\times 200)$. Bar $=50 \mu \mathrm{m}$. e: The microvessel density (MVD) in Endostar-treated mice was markedly lower than that in the control group $(P<0.05)$. f: The microlymphatic vessel density (MLD) in the Endostar group was markedly lower than that in control group $(P<0.05)$

\section{Discussion}

Nowadays, colorectal cancer is one of the most common causes of malignant tumor mortality worldwide. Most of these deaths occur in patients with locally advanced colorectal 


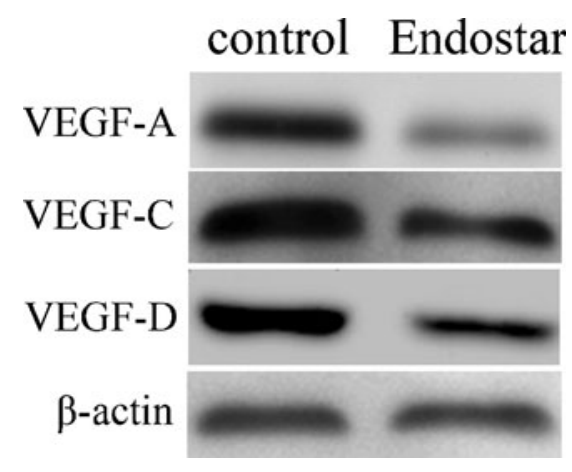

Fig. 4 VEGF-A, -C, and -D protein expression levels in SW620 cells detected by Western blotting analysis. Endostar downregulated the expression of VEGF-A,-C,-D in SW620 cells in vitro

cancer. Although both the incidence and death rate for colorectal cancer has been decreasing over the previous two decades in Western countries, its incidence rate is on the rise in the Eastern hemispheres [25, 26]. Unfortunately the common therapeutic regimen for colorectal cancer, including surgery, chemotherapy and radiation, are insufficient to eliminate the tumor burden and colorectal cancer continues to inflict a heavy toll worldwide. Therefore, there is an urgent need to develop novel therapeutic approaches for combating this devastating disease.

Angiogenesis is essential for the development, growth and advancement of solid tumors, including those associated with colorectal cancer. Recently, anti-angiogenic therapy has emerged as a treatment of malignant disease [27]. However, the genomic instability and heterogeneity of tumor cell populations lead to a shift of the expression of angiogenic factors during tumor progression. Various tumors at their advanced stage express multiple angiogenic factors [5]. At this stage, anti-VEGF-A monotherapy such as bevacizumab would be predicted to be ineffective or even encounter drug resistance. Furthermore, anti-VEGF-A monotherapy might even help tumors to switch on other angiogenic pathways for angiogenesis [28]. As a result, tumors can escape from antiVEGF-A therapy after a relative long-term treatment [29]. Additionally, these drugs can increase some side effects such as rash, diarrhea, hypertension, even gastrointestinal perforation and arterial thromobembolic events [7, 8]. It is therefore necessary to find the new target therapy to treat colorectal cancer.

Endostatin is another important angiogenesis inhibitor, and it is one of the most potent of the known inhibitors of tumor angiogenesis. Many published reports showed that endostatin mainly suppresses pathological angiogenesis by downregulating a number of angiogenic factors, including VEGF-A and FGF-2, and produces virtually no toxicity after long-term delivery [30, 31]. Endostar is a novel recombinant human endostatin. The current data revealed that Endostar exerts anti-angiogenic effects via a similar mechanism as endostatin. However, Endostar differs from endostatin in that it has an additional nine-amino acid sequence with zinc binding activity that increases Endostar's half-life [32].

Using the noninvasive Xenogen in vivo imaging system, we found that Endostar inhibited not only tumor growth, but also lymph node metastasis in a mouse xenograft model, which was confirmed by pathological examination at the end of the experiment. This imaging system relies on the measurement of luminescence produced by tumor cells engineered to express luciferase, which is used as a way to evaluate viability of tumor cells. Thus, the Xenogen system provides a more accurate indication of anti-tumor activity and anti-metastasis effect of Endostar than measurement of tumor volume or the examination of the axilla and groin area lymph nodes [33].

Previous studies have demonstrated that lymph node metastasis is an independent prognostic factor for malignant tumors [34, 35]. However, it is difficult to prevent lymph node metastasis completely using the current treatments for colorectal cancer. Our findings indicated that Endostar may reduce the possibility of tumor cells entering into the lymph nodes. Endostar should ideally be administered at early stage in order to reduce the spread of colorectal cancer cells to regional lymph node, i.e. before the induction of lymphangiogenesis in tumor progression.

In order to elucidate the mechanism whereby Endostar inhibited lymphangiogenesis, we investigated the effect of Endostar on the expression of VEGF-C and VEGF-D in vitro. We showed that Endostar suppressed the expression of these factors. However, this result did not agree with findings from previous studies completely, which demonstrated that endostatin inhibits lymphangiogenesis and lymph node metastasis by downregulating VEGF-C rather than VEGF-D expression in gastric cancer or skin tumor tissues [17, 18]. Therefore, Endostar maybe differ from endostatin in regulating lymphangiogenesis in different types of tumors. It has been reported that both VEGF-C and VEGF-D are upregulated in colorectal cancer, and both of them not only increase the density but also induce the enlargement of lymphatic vessels, which leads to metastasis to regional lymph nodes [14]. Although the role of VEGF-D in lymphangiogenesis is still relatively inconclusive, most researchers believe that VEGF-D is at least a predictor for lymphatic metastasis, or an independent prognostic factor $[36,37]$.

In summary, Endostar inhibits not only angiogenesis, but also lymphangiogenesis and lymph node metastasis, by inhibiting the expressions of VEGF-A, VEGF-C and VEGF-D in colorectal cancer. Our findings thus provide a new strategy to develop novel treatments for colorectal cancer. 
Acknowledgements We thank Dr. James S. Martin (School of Medicine, University of Pennsylvania) for preparing the article. We also thank Dr. Jingguo You for the technical assistance. This research was supported by Natural Science Foundation of Hebei province of China (05547008D-3).

Competing interests We declare that we have no competing interests.

Open Access This article is distributed under the terms of the Creative Commons Attribution Noncommercial License which permits any noncommercial use, distribution, and reproduction in any medium, provided the original author(s) and source are credited.

\section{References}

1. Center MM, Jemal A, Ward E (2009) International trends in colorectal cancer incidence rates. Cancer Epidemiol Biomarkers Prev 6:1688-1694

2. Becouarn Y, Rougier P (1998) Clinical efficacy of oxaliplatin monotherapy: phase II trials in advanced colorectal cancer. Semin Oncol 25(2 Suppl 5):23-31

3. Ng M, Roy-Chowdhury S, Lum SS, Morgan JW, Wong JH (2009) The impact of the ratio of positive to total lymph nodes examined and outcome in colorectal cancer. Am Surg 75:873-876

4. Jemal A, Clegg LX, Ward E, Ries LA, Wu X, Jamison PM, Wingo PA, Howe HL, Anderson RN, Edwards BK (2004) Annual report to the nation on the status of cancer, 1975-2001, with a special feature regarding survival. Cancer 101:3-27

5. Folkman J (2002) Role of angiogenesis in tumor growth and metastasis. Semin Oncol 29(6 Suppl 16):15-18

6. Byrne AM, Bouchier-Hayes DJ, Harmey JH (2005) Angiogenic and cell survival functions of vascular endothelial growth factor (VEGF). J Cell Mol Med 9:777-794

7. Okines A, Puerto OD, Cunningham D, Chau I, Van Cutsem E, Saltz L, Cassidy J (2009) Surgery with curative-intent in patients treated with first-line chemotherapy plus bevacizumab for metastatic colorectal cancer First BEAT and the randomised phase-III NO16966 trial. Br J Cancer 101:1033-1038

8. Hsu JY, Wakelee HA (2009) Monoclonal antibodies targeting vascular endothelial growth factor: current status and future challenges in cancer therapy. BioDrugs 23:289-304

9. Punt CJ, Tol J (2009) More is less-combining targeted therapies in metastatic colorectal cancer. Nat Rev Clin Oncol 6:731-733

10. Duff SE, Jeziorska M, Kumar S, Haboubi N, Sherlock D, O'Dwyer ST, Jayson GC (2007) Lymphatic vessel density, microvessel density and lymphangiogenic growth factor expression in colorectal cancer. Colorectal Dis 9:793-800

11. Royston D, Jackson DG (2009) Mechanisms of lymphatic metastasis in human colorectal adenocarcinoma. J Pathol 217:608-619

12. Sundlisaeter E, Dicko A, Sakariassen PØ, Sondenaa K, Enger PØ, Bjerkvig R (2007) Lymphangiogenesis in colorectal cancer-prognostic and therapeutic aspects. Int J Cancer 121:1401-1409

13. Onogawa S, Kitadai Y, Tanaka S, Kuwai T, Kimura S, Chayama $\mathrm{K}$ (2004) Expression of VEGF-C and VEGF-D at the invasive edge correlates with lymph node metastasis and prognosis of patients with colorectal carcinoma. Cancer Sci 95:32-39

14. Duff SE, Li C, Jeziorska M, Kumar S, Saunders MP, Sherlock D, O'Dwyer ST, Jayson GC (2003) Vascular endothelial growth factors $\mathrm{C}$ and $\mathrm{D}$ and lymphangiogenesis in gastrointestinal tract malignancy. Br J Cancer 89:426-430

15. Achen MG, Mann GB, Stacker SA (2006) Targeting lymphangiogenesis to prevent tumour metastasis. Br J Cancer 94:1355-1360
16. Wissmann C, Detmar M (2006) Pathways targeting tumor lymphangiogenesis. Clin Cancer Res 12:6865-6868

17. Brideau G, Mäkinen MJ, Elamaa $\mathrm{H}, \mathrm{Tu} \mathrm{H}$, Nilsson G, Alitalo K, Pihlajaniemi T, Heljasvaara R (2007) Endostatin overexpression inhibits lymphangiogenesis and lymph node metastasis in mice. Cancer Res 67:11528-11535

18. Fukumoto S, Morifuji M, Katakura Y, Ohishi M, Nakamura S (2005) Endostatin inhibits lymph node metastasis by a down-regulation of the vascular endothelial growth factor $\mathrm{C}$ expression in tumor cells. Clin Exp Metastasis 22:31-38

19. Zhuo W, Luo C, Wang X, Song X, Fu Y, Luo Y (2010) Endostatin inhibits tumour lymphangiogenesis and lymphatic metastasis via cell surface nucleolin on lymphangiogenic endothelial cells. J Pathol 222:249-260

20. Feldman AL, Alexander HR Jr, Bartlett DL, Kranda KC, Miller MS, Costouros NG, Choyke PL, Libutti SK (2001) A prospective analysis of plasma endostatin levels in colorectal cancer patients with liver metastases. Ann Surg Oncol 8:741-745

21. Chen W, Fu J, Liu Q, Ruan C, Xiao S (2003) Retroviral endostatin gene transfer inhibits human colon cancer cell growth in vivo. Chin Med J (Engl) 116:1582-1584

22. te Velde EA, Reijerkerk A, Brandsma D, Vogten JM, Wu Y, Kranenburg O, Voest EE, Gebbink M, Borel Rinkes IH (2005) Early endostatin treatment inhibits metastatic seeding of murine colorectal cancer cells in the liver and their adhesion to endothelial cells. Br J Cancer 92:729-735

23. Yang L, Wang JW, Sun Y, Zhu YZ, Liu XQ, Li WL (2006) Randomized phase II trial on escalated doses of Rh-endostatin (YH-16) for advanced non-small cell lung cancer. Zhonghua Zhong Liu Za Zhi 28:138-141

24. Zhang DW, Li HL, Yao Q, Yang WL, Wang HL, Zhai DX, Zhou ZH (2010) The synergistic effect of recombinant human endostatin (YH-16) combined with oxaliplatin on human colorectal carcinoma. J Int Med Res 38:111-126

25. La Vecchia C, Bosetti C, Lucchini F, Bertuccio P, Negri E, Boyle P, Levi F (2010) Cancer mortality in Europe, 2000-2004, and an overview of trends since 1975. Ann Oncol 21:1323-1360

26. Jung KW, Won YJ, Park S, Kong HJ, Sung J, Shin HR, Park EC, Lee JS (2009) Cancer statistics in Korea: incidence, mortality and survival in 2005. J Korean Med Sci 24:995-1003

27. Fujita Y, Abe R, Shimizu H (2008) Clinical approaches toward tumor angiogenesis: past, present and future. Curr Pharm Des $14: 3820-3834$

28. Ferrara N, Kerbel RS (2005) Angiogenesis as a therapeutic target. Nature 438:967-974

29. Casanovas O, Hicklin DJ, Bergers G, Hanahan D (2005) Drug resistance by evasion of antiangiogenic targeting of VEGF signaling in late-stage pancreatic islet tumors. Cancer Cell 8:299-309

30. Abdollahi A, Hahnfeldt P, Maercker C, Gröne HJ, Debus J, Ansorge W, Folkman J, Hlatky L, Huber PE (2004) Endostatin's antiangiogenic signaling network. Mol Cell 13:649663

31. Akbulut H, Altuntas F, Akbulut KG, Ozturk G, Cindoruk M, Unal E, Icli F (2002) Prognostic role of serum vascular endothelial growth factor, basic fibroblast growth factor and nitric oxide in patients with colorectal carcinoma. Cytokine 20:184-190

32. Ling Y, Yang Y, Lu N, You QD, Wang S, Gao Y (2007) Endostar, a novel recombinant human endostatin, exerts antiangiogenic effect via blocking VEGF-induced tyrosine phosphorylation of KDR/Flk-1 of endothelial cells. Biochem Biophys Res Commun 361:79-84

33. Jenkins DE, Yu SF, Horning YS, Purchio T, Contag PR (2003) In vivo monitoring of tumor relapse and metastasis using bioluminescent PC-3M-luc-C6 cells in murine models of human prostate cancer. Clin Exp Metastasis 20:745-756 
34. Lee HY, Choi HJ, Park KJ, Shin JS, Kwon HC, Roh MS, Kim C (2007) Prognostic significance of metastatic lymph node ratio in node-positive colon carcinoma. Ann Surg Oncol 14:1712-1717

35. Bock F, Onderka J, Dietrich T, Bachmann B, Kruse FE, Paschke M, Zahn G, Cursiefen C (2007) Role of lymphangiogenesis in cancer. J Clin Oncol 25:4298-4307

36. Thelen A, Scholz A, Benckert C, von Marschall Z, Schröder M, Wiedenmann B, Neuhaus P, Rosewicz S, Jonas S (2008)
VEGF-D promotes tumor growth and lymphatic spread in a mouse model of hepatocellular carcinoma. Int $\mathrm{J}$ Cancer 122:2471-2481

37. Kopfstein L, Veikkola T, Djonov VG, Baeriswyl V, Schomber T, Strittmatter K, Stacker SA, Achen MG, Alitalo K, Christofori G (2007) Distinct roles of vascular endothelial growth factor-D in lymphangiogenesis and metastasis. Am J Pathol 170:1348-1361 